\title{
Exciting new article categories introduced not Clinical and Experimental Metastasis: submit your article now!
}

\author{
Jörg Haier ${ }^{1}\left[\right.$ D J Jonathan P. Sleeman ${ }^{2,3}$ \\ Received: 29 January 2018 / Accepted: 8 February 2018 / Published online: 7 March 2018 \\ (c) Springer Science+Business Media B.V., part of Springer Nature 2018
}

The Editors-in-Chief of Clinical and Experimental Metastasis have set themselves the task of making the journal the premier choice for publishing cutting edge metastasis research. As the official journal of the Metastasis Research Society, we are also aware of the importance that Clinical and Experimental Metastasis develops to serve the needs and interests of the whole breadth of the community working on the problem of metastasis. In particular, we want to expand the exposure and reputation of the journal amongst clinical researchers. We also want the journal to become a forum for young investigators to share their ideas, and which helps them establish themselves in the metastasis research community. In addition, we aim to develop the journal not only as a platform for publishing excellent metastasis research, but also as a resource that supports investigators in their research activities. To achieve these aims, we have decided to implement four new types of articles that will be published in the journal, in addition to established article types such as original research and in-depth review articles. These new article categories are outlined below.

\section{Clinical and Translational Perspectives}

Clinical and Translational Perspectives provide a concise and comprehensive overview of current aspects of clinical and translational metastasis research. These articles

Jörg Haier

joerg.haier@nordakademie.de

1 NORDAKADEMIE University of Applied Science, Köllner Chaussee 11, 25337 Elmshorn, Germany

2 University of Heidelberg, Medical Faculty Mannheim, CBTM, Ludolf-Krehl-Str. 13 - 17, 68167 Mannheim, Germany

3 Karlsruhe Institute for Technology (KIT), Campus Nord, Institut für Toxikologie und Genetik, Postfach 3640, 76021 Karlsruhe, Germany address unsolved problems and unmet needs that have a high importance for the care of patients with metastatic cancer, and which need to be targeted by clinical or translational research.

Articles should have a length of about 2000-3000 words with an unlimited number of tables and figures, and a limit of about 30 references. Manuscripts should include

- A description of the clinical setting/patient group

- A description of the unsolved clinical problem

- An overview of the current status of the bench-to-bedside or bedside-to-bench transition

- Hypotheses and questions that need to be answered by future experimental research

\section{Rising Star Reviews}

Rising Star Reviews gives graduate students and postdocs the chance to publish a review article on a metastasis-related topic. This is a chance, for example, for thesis introductions to be converted into a review article, or for literature research performed when starting work in a new field to be leveraged in the form of a publication. It is also a chance for young investigators to air new ideas and hypotheses, and to gain exposure in the metastasis research community. Mentoring in the writing of review articles is expected, and can be provided either by the author's supervisor, or by a member of the editorial board. No word, reference or figure limits are specified for these reviews, but authors are advised that concise scientific writing is expected. Individuals interested in submitting a Rising Star Review are invited to contact one of the Editors-in-Chief with an abstract outlining the proposed article, together with details about mentoring that is in place or is required. 


\section{Technical Notes in Metastasis Research}

Technical Notes in Metastasis Research summarise research approaches and methodology that is used in the investigation of metastasis. Articles may provide a comprehensive overview of experimental design and a step-by-step description of methods, for example in the form of an experimental protocol. They may also describe current quality standards that need to be considered when using particular techniques, or suggest news standards that need to be implemented by the community. This type of article may also be used to describe new methods that can be employed in metastasis research.

This type of article has a maximum length of about 3000 words, with an unlimited number of tables and figures and a limit of about 50 references. As appropriate, in addition to a brief abstract (maximum 150 words), manuscripts should include

- A description of the applications that the technique can be used for

- Principles of the methodology, including if appropriate a detailed experimental protocol

- Hints and tips: advice and suggestions concerning the practicalities of carrying out the experimental technique and optimizing its performance

- Advantages and limitations of the methodology

- Recommendations for data validation and quality control

\section{Molecules in Metastasis}

Molecules in Metastasis articles provide a concise overview of the current knowledge about specific molecules that play a functional role in cancer metastasis in the form of a mini review. In contrast to full review articles, these articles provide a brief and comprehensive introduction into the metastasis-relevant molecular biology and cellular functions of a single or small family of molecules, as well as their clinical importance as appropriate. These articles should be easy to read and assimilate, and should provide an authoritative primer for those new to the field. Emphasis is placed on brevity and conciseness in these articles.

This type of article should usually fit into a maximum of four journal pages (circa 2500 words) with a maximum of 3 tables and figures and an upper limit of about 70 references. Manuscripts should include information about

- Domain structure of the protein(s) and structural aspects as appropriate

- Physiological molecular and cellular functions

- Specific functional roles in cancer metastasis biology

- Potential as a predictive, prognostic or therapeutic target

The Editors-in-Chief of Clinical and Experimental Metastasis are extremely excited about these new innovations in the journal. Please consider submitting one of these new article types in the near future. In addition, we strongly encourage the metastasis research community to submit your best research - be that basic or clinical - to the journal. We also continue to warmly welcome suggestions for in-depth review articles, as well as initiatives from individuals interested in acting as Guest Editors who want to set up a special thematic issue. We look forward to hearing from you! 